\title{
Psychobiotics for Patients with Chronic Gastrointestinal Disorders Having Anxiety or Depression Symptoms
}

\author{
Viet Hang Dao (1D ${ }^{1,2}$ \\ Long Bao Hoang (D) ${ }^{2}$ \\ Thi Oanh Trinh ${ }^{2}$ \\ Thi Thu Trang Tran ${ }^{2}$ \\ Van Long Dao ${ }^{1,2}$
}

'Internal Medicine Faculty, Hanoi Medical University, Hanoi, Vietnam; ${ }^{2}$ Research and Training Management Unit, Institute of Gastroenterology and Hepatology, Hanoi, Vietnam
Correspondence: Viet Hang Dao Email daoviethang@hmu.edu.vn
Purpose: Using psychobiotics to modify the gut microbiome has been shown to improve the anxiety and depression situation of patients with chronic gastrointestinal (GI) symptoms. This study evaluated changes in depression, anxiety, GI symptomss and side effects when patients used a multispecies probiotics product.

Patients and Methods: A single-center uncontrolled trial was conducted in patients with chronic GI symptoms, anxiety and depression who used a multispecies probiotics product. The patients were screened for anxiety and depression symptoms using the Hospital Anxiety and Depression Scale (HADS). Those who had a component score of 8 or higher were given the multispecies probiotics product for 2 months and followed up after 1 and 2 months. All data are collected and managed in a case report form.

Results: Eighty-three patients were enrolled, with a mean age (SD) of 43.9 (12.3) years; $73.5 \%$ of the patients were female. Of these patients, 8 met the Rome IV criteria for irritable bowel syndrome. The HADS scores displayed significant improvement at follow-up. The mean (SD) total HADS scores were 20.0 (6.3), 7.2 (5.4), and 4.9 (5.1) at baseline, 1 month, and 2 months, respectively. Quality of life also improved significantly. A small proportion $(<5 \%)$ of patients developed mild symptoms, including fullness, diarrhea, and sleep complaints.

Conclusion: After 2 months using the probiotic product, the symptoms of anxiety and depression improved significantly. Mild gastrointestinal or constitutional symptoms developed in some patients.

Keywords: probiotics, anxiety, depression

\section{Introduction}

Anxiety and depression are present in 30-50\% of patients with chronic gastrointestinal (GI) symptoms ${ }^{1-4}$ and are thought to be associated with the gut-brain axis (GBA), which is the bidirectional signal pathway between the central nervous system and the enteric nervous system. ${ }^{5-7}$ Evidence has shown that the GBA and the gut microbiome interact in both healthy people and patients with inflammatory bowel disease, irritable bowel syndrome (IBS), obesity, cardiovascular diseases, and depression. $^{8-11}$ The gut microbiota is postulated to interact with the GBA via three pathways: stimulating the intestinal epithelium to secrete neuroactive molecules, affecting serum cytokine levels, and directly activating the vagus nerve. ${ }^{6,12}$ Therefore, modifying the gut microbiome, which in turn modifies the activity of the GBA, is expected to improve the symptoms of anxiety and depression. In 2013, the 
term "psychobiotics" has been defined as the novel class of probiotics, which are living microorganisms that provide health benefits to the host when used in appropriate amounts. ${ }^{13}$ This perspective has led to the use of probiotics as a supportive therapy in psychiatric diseases including anxiety and depression. ${ }^{14-17}$ The evidence of efficacy in improving some diseases such as functional gastrointestinal disorders as well as in stress, anxiety and depression have been reported for some Lactobacillus, Lactococcus and Bifidobacterium strains. ${ }^{18,19}$ Some previous studies have shown that multispecies probiotics which is the combination of different strains of specific genera may increase effectiveness through an additive effect of specific strain properties. ${ }^{20-22}$

In Vietnam, mental disorders have increased in recent years. Depression and anxiety are the second and third most common mental disorders, with the prevalence being $2.8 \%$ and $2.6 \%$, respectively. ${ }^{23}$ The prevalence in the elderly was much higher $(26.4 \%) .^{24}$ Diabetes and rheumatic diseases are associated with a higher prevalence of anxiety and depression. ${ }^{25,26}$ In one study on IBS in Vietnamese pharmacy students, the prevalence of anxiety and depression among IBS people were $62.5 \%$ and $45.5 \%$, respectively, and the prevalence of anxiety was significantly higher than in the control group. ${ }^{27}$ This study also reported a correlation between canned food and IBS, which raises the question about the relationship between diet, microbiota and the occurrence of IBS. ${ }^{27}$ However, data on the effectiveness of multispecies probiotics in alleviating anxiety and depression symptoms among patients with chronic GI symptoms are still lacking in the world and in Vietnam.

Therefore, we conducted this study to evaluate changes in depression, anxiety, GI symptoms and side effects that developed when patients used a multispecies probiotics product.

\section{Patients and Methods Study Population}

We conducted a single-center uncontrolled trial on patients who had symptoms suggesting anxiety/depression but were not clinically diagnosed with either condition at Hoang Long Clinic and Institute of Gastroenterology and Hepatology from January 2019 to January 2020.

First, we conducted anxiety/depression screening for all patients visiting our study site for health checkup who were over 18 years old, had chronic GI symptoms over $>3$ months and had no suspected lesions of malignancy or acute inflammation on a current endoscopy. Anxiety/depression screening was done using the Hospital Anxiety and Depression Scale (HADS), which was originally designed for general hospital settings. ${ }^{28}$ The HADS includes seven questions assessing depression and seven assessing anxiety. Each question is answered on a scale of 0 to 3 . The total scores for the depression and anxiety components are calculated, with each component score ranging from 0 to $21 .^{28,29}$ A depression/anxiety component score of 8 or higher suggests possible depression/anxiety, respectively. ${ }^{30,31}$

Then, those who had a component score of 8 or higher were invited to participate in the study. Patients who agreed to participate would receive an 8-week multispecies probiotic supplementation in addition to the medications prescribed by the clinic's doctor. The prescribed medications might include antibiotics (often for the treatment of Helicobacter pylori or small intestinal bacterial overgrowth), proton-pump inhibitors, or other medications for gastrointestinal conditions. Psychiatric drugs were not prescribed for our study patients.

The multispecies probiotic product contains eight bacterial strains including Bifidobacterium bifidum W23, Bifidobacterium lactis W52, Lactobacillus acidophilus W37, Lactobacillus brevis W63, Lactobacillus casei W56, Lactobacillus salivarius W24, Lactococcus lactis W19, and Lactococcus lactis W58. The product consists of over 2,5 x $10^{9}$ CFU per gram with other excipients including corn starch, maltodextrins, potassium chloride, magnesium sulphate, manganese sulphate, inulin and plant-based proteins. Dosage form is the dry powder which be supplied as sachets. This product was manufactured in Netherlands by Winclove B.V Company and distributed in Vietnam by Dong Do Pharmacy Company. It has been approved by the Department of Food Safety, Vietnam's Ministry of Health for the use as a supplement in patients with symptoms of depression and/or anxiety (approval No. 38162/2017/ATTP-XNCB). Patients were instructed to use the multispecies probiotic product with their prescribed medications before meal. Patients would mix one sachet ( $2 \mathrm{~g}$ of product) in $100 \mathrm{~mL}$ of water, let stand for 1 minute, and stir well before use.

Patients were followed up after 1 and 2 months via phone interviews. The analytic population included only patients who had finished 2-month follow-up.

\section{Data Collection}

All participants were asked about their medical history, presenting GI symptoms (regurgitation, epigastric pain, 
heartburn, nausea, dysphagia, vomiting, changes in bowel habits, constipation, diarrhea, fecal incontinence) and constitutional symptoms, and quality of life assessed by the European Quality of Life scale EQ-5D-5L. ${ }^{32,33}$ On 1- and 2-month follow-up phone interviews, they were asked about current depression/anxiety status (HADS), quality of life (EQ-5D-5L), whether their presenting symptoms had changed (improved/not changed/worsened), and whether they had any new symptoms developed while using the multispecies probiotics product (yes/no questions). Patients were encouraged to share any complaints or issues with taking the prescribed medications and the multispecies probiotics product.

\section{Statistical Analysis}

Demographic characteristics, clinical symptoms, medical history of the study participants are presented as numbers and percentages. The HADS and EQ-5D-5L scores are presented as the mean (standard deviation) or median (interquartile range). To compare between baseline, 1 month and 2 months, the Cochran's Q test was used to evaluate the changes in participants' symptoms and repeated-measures analysis of variance (RM-ANOVA) was used to compare the differences in the HADS and EQ-5D-5L scores. Mauchly's sphericity test was performed to check the assumption of sphericity, which is critical for RM-ANOVA, and Greenhouse-Geisser correction was applied if the assumption is violated. Differences were considered statistically significant when the p-value was $<0.05$. All statistical analyses were performed using the Python programming language.

\section{Ethical Consideration}

The study was conducted in accordance with the Declaration of Helsinki and approved by the Institutional Review Board of the Dinh Tien Hoang Institute of Medicine under decision No. IRB-1908. All study participants were given all information about the study's objectives and process and provided informed consents to sign in before the study started. All study participants voluntarily participated in this study.

\section{Results}

A total of 202 patients participated in the anxiety/depression screening in Hoang Long clinic between January 2019 and January 2020, 111 of whom were eligible and had been given the probiotics. We excluded 28 patients due to loss to follow-up (18 patients) or discontinuing the product (10 patients). Among them, 7 patients felt that the product was not effective ( 7 patients), 2 patients were being treated for other diseases (2 patients), and 1 patient was pregnant. Eventually, 83 eligible patients were included in our analysis. The baseline characteristics of the subjects are presented in Table 1 .

\section{Changes in HADS and Quality of Life Scores}

The HADS score (both the total score and the component scores) and the EQ-5D-5L score improved significantly after 1 month and continued to improve after 2 months of using the multispecies probiotics. The mean (SD) HADS score decreased from 20.0 (6.3) to 7.2 (5.4) and 4.9 (5.1), and the mean (SD) EQ-5D-5L score increased from $0.67(0.12)$ to $0.82(0.08)$ and $0.85(0.09)$ after 1 and 2 months, respectively. All differences were statistically significant $(\mathrm{p}<0.001)$ (Figure 1$)$.

Table I Baseline Characteristics of the Subjects

\begin{tabular}{|l|c|}
\hline Characteristics & Number(\%) $^{\mathbf{a}}$ \\
\hline Sex & $61(73.5)$ \\
Male & $22(26.5)$ \\
\hline Age (years) & $43.9(12.3)$ \\
\hline Diagnosis at baseline & \\
IBS (Rome IV) & $8(9.6)$ \\
\hline Upper gastrointestinal lesions & \\
Barrett's esophagus & $3(3.6)$ \\
Reflux esophagitis & $34(41.0)$ \\
Chronic gastritis & $67(80.7)$ \\
Duodenitis & $5(6.0)$ \\
\hline Lower gastrointestinal lesions & \\
Colorectal polyp & $5(6.0)$ \\
Colitis & $3(3.6)$ \\
Internal hemorrhoids & $31(37.4)$ \\
\hline Past symptoms of anxiety/depression & $19(22.9)$ \\
\hline HADS and EQ-5D-5L score & \\
\hline HADS (total) & $0.67(0.12)$ \\
HADS (anxiety) & $11.0(3.8)$ \\
HADS (depression) & $9.0(3.8)$ \\
EQ-5D-5L & \\
\hline Non & \\
\hline
\end{tabular}

Notes: ${ }^{a}$ Categorical data are presented as the number (percentage), quantitative data are presented as the mean (standard deviation). 

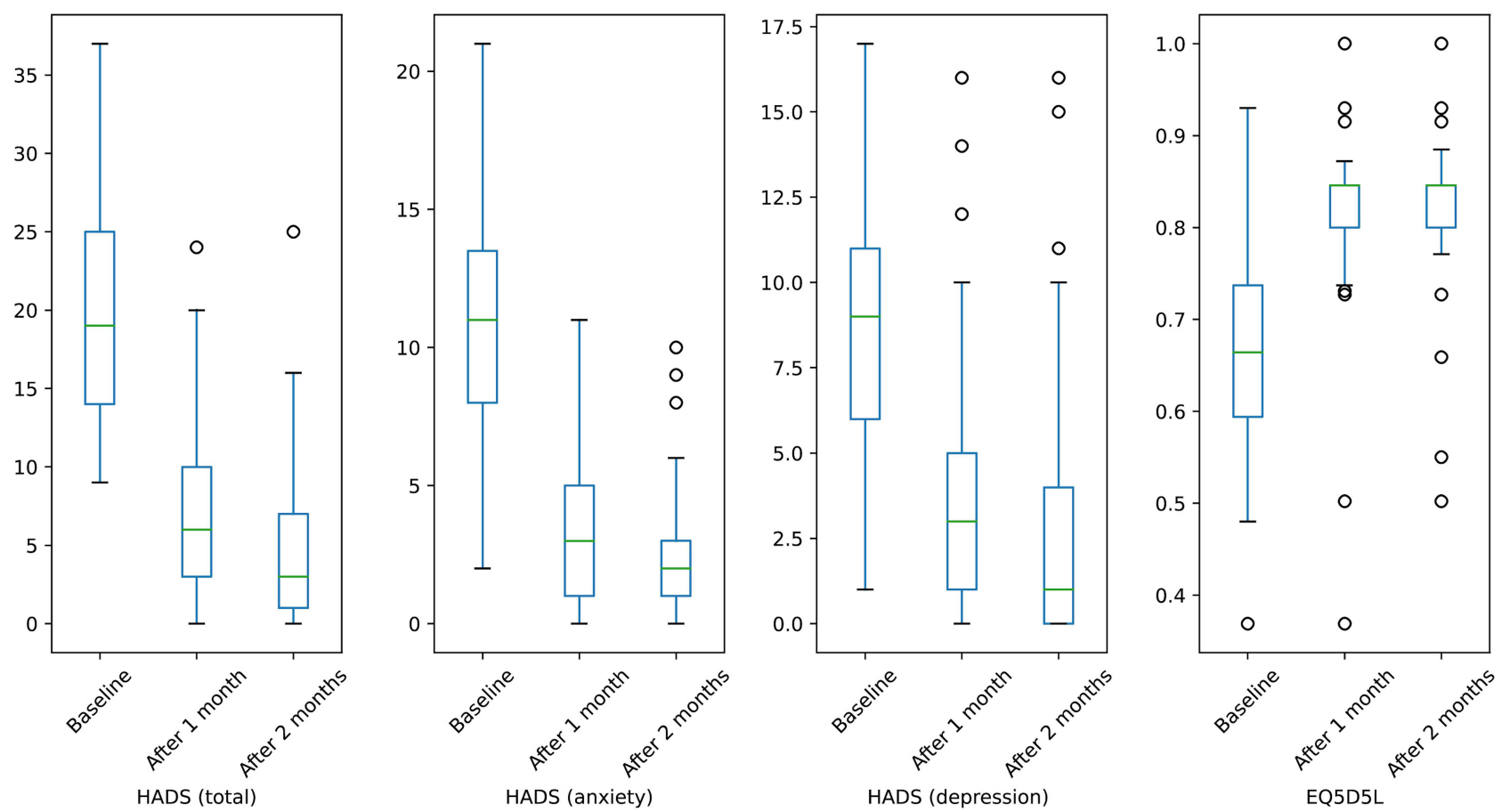

Figure I Changes of HADS and EQ5D5L score before and after using the multispecies probiotics product.

\section{Changes in Baseline GI Symptoms}

Table 2 presents the changes in baseline GI symptoms after 1 and 2 months using this multispecies probiotics product. The proportion of symptomatic patients (symptom-wise) decreased significantly after 1 month and continued to decrease after 2 months.

\section{New Symptoms While Using the Product}

Some patients developed new symptoms while using the product; these symptoms were mild and uncommon $(<5 \%)$. Notably, one patient described a burning sensation in the throat ten days after starting using the product and had to stop using it after the first month. We followed up this patient at 2 months and the symptom completely resolved.

\section{Discussion}

In this study, we examined patients with chronic GI symptoms who also had symptoms of anxiety and/or depression. Anxiety and depression are common presentations in patients with functional GI disorders. Hartono et al. reported that anxiety and/or stress were present in $20-60 \%$ of patients with IBS, non-erosive reflux disease, and functional dyspepsia. ${ }^{34}$ The prevalence of depression and anxiety in IBS patients was reported to be higher than in healthy controls in some case-control studies (pooled difference 33\% and $19 \%$, respectively). ${ }^{35}$ In some previous Vietnamese studies, anxiety and depression were present in up to $60 \%$ of IBS patients. ${ }^{27,36}$ However, only $9.6 \%$ of our patients fulfilled the Rome IV criteria for IBS. This suggests that anxiety and depression might be more common even outside the domain of functional GI disorders, emphasizing the importance of screening and managing these neuropsychiatric symptoms in patients with GI disorders in general.

According to the modern perspective on the links between the gut microbiome, the GBA, and psychiatric symptoms, it is possible that GI patients with anxiety or depression might benefit from using probiotics to modify the gut microbiome. Our study investigated the changes in anxiety and depression symptoms in patients when taking the probiotics that contains eight Bifidobacterium and Lactobacillus strains. This probiotics product could be categorized as a psychobiotic due to the efficacy of specific probiotics strains which have been reported an improvement in some psychiatric diseases as well as mood for patients. ${ }^{20,21,37,38}$ The HADS score (both the total and component scores) decreased significantly at 1 and 2 months, implying a significant improvement in anxiety and depression symptoms. The effectiveness of probiotics on depression was also demonstrated in 2 clinical trials on IBS patients by PintoSanchez et al (Bifidobacterium longum NCC3001) ${ }^{39}$ and Lorenzo-Zuniga et al (a combination of 3 Bifidobacterium and Lactobacillus strains). ${ }^{22}$ Furthermore, some studies on 
Table 2 Symptom Changes After I Month and 2 Months

\begin{tabular}{|c|c|c|c|c|c|c|}
\hline Symptom n (\%) & Baseline & After I Month & After 2 Months & p value ${ }^{b}$ & $\begin{array}{l}\text { New Onset } \\
\text { During the } \\
\text { Ist Month }\end{array}$ & $\begin{array}{l}\text { New Onset } \\
\text { During the } \\
\text { 2nd Month }\end{array}$ \\
\hline \multicolumn{7}{|l|}{ Upper Gl symptoms } \\
\hline Regurgitation & $60(72.3)$ & $13(15.7)$ & $10(12.1)$ & $<0.001$ & - & - \\
\hline Epigastric pain & $43(5 \mid .8)$ & II (I3.3) & $\mathrm{I}(\mathrm{I} .2)$ & $<0.001$ & - & - \\
\hline Heartburn & $37(44.6)$ & $6(7.2)$ & $5(6.0)$ & $<0.001$ & $\mathrm{I}(\mathrm{I} .2)$ & - \\
\hline Nausea & $29(34.9)$ & $2(2.4)$ & $\mathrm{I}(\mathrm{I} .2)$ & $<0.001$ & $2(2.4)$ & - \\
\hline Dysphagia & $26(31.3)$ & $9(10.8)$ & $6(7.2)$ & $<0.001$ & - & - \\
\hline Vomiting & $5(6.0)$ & $0(0.0)$ & $0(0.0)$ & 0.007 & - & - \\
\hline \multicolumn{7}{|l|}{ Lower Gl symptoms } \\
\hline Change in bowel habits & $35(42.2)$ & $10(12.1)$ & $4(4.8)$ & $<0.001$ & - & - \\
\hline Constipation & $23(27.7)$ & $14(16.9)$ & $9(10.8)$ & 0.005 & - & - \\
\hline Diarrhea & $13(15.7)$ & $4(4.8)$ & $3(3.6)$ & $<0.001$ & $4(4.8)$ & - \\
\hline Fecal incontinence & $9(10.8)$ & $\mathrm{I}(\mathrm{I} .2)$ & $\mathrm{I}(\mathrm{I} .2)$ & $<0.001$ & - & - \\
\hline \multicolumn{7}{|l|}{ Non-specific symptoms } \\
\hline Fatigue & $65(78.3)$ & $12(14.5)$ & $4(4.8)$ & $<0.001$ & - & - \\
\hline $\begin{array}{l}\text { Abdominal pain (other } \\
\text { regions) }\end{array}$ & $56(67.5)$ & $10(12.1)$ & $3(3.6)$ & $<0.001$ & $\mathrm{I}(1.2)$ & - \\
\hline Loss of appetite & $49(59.0)$ & $10(12.1)$ & $5(6.0)$ & $<0.001$ & - & - \\
\hline Fullness & $48(57.8)$ & $7(8.4)$ & $2(2.4)$ & $<0.001$ & $4(4.8)$ & - \\
\hline Bloating & $48(57.8)$ & $9(10.8)$ & $7(8.4)$ & $<0.001$ & - & - \\
\hline Weight loss & $40(48.2)$ & $19(22.9)$ & $12(14.5)$ & $<0.001$ & - & - \\
\hline Dyspepsia & $40(48.2)$ & $8(9.6)$ & I (I.2) & $<0.001$ & - & - \\
\hline Globus & $33(39.8)$ & $8(9.6)$ & $7(8.4)$ & $<0.001$ & - & - \\
\hline Chest pain & $26(3 \mid .3)$ & $5(6.0)$ & $3(3.6)$ & $<0.001$ & - & - \\
\hline Sleep complaints & $0(0.0)$ & $3(3.6)$ & $\mathrm{I}(\mathrm{I} .2)$ & 0.1 & $3(3.6)$ & $\mathrm{I}(\mathrm{I} .2)$ \\
\hline Dizziness & $0(0.0)$ & $\mathrm{I}(\mathrm{I} .2)$ & $0(0)$ & 0.37 & $\mathrm{I}(\mathrm{I} .2)$ & - \\
\hline Headache & $0(0.0)$ & I (I.2) & $0(0)$ & 0.37 & $\mathrm{I}(\mathrm{I} .2)$ & - \\
\hline $\begin{array}{l}\text { Burning sensation in the } \\
\text { throat }\end{array}$ & $0(0.0)$ & I (I.2) & $0(0)$ & 0.37 & $\mathrm{I}(\mathrm{I} .2)$ & - \\
\hline
\end{tabular}

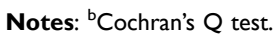

both healthy people and depressed patients that used different probiotic products demonstrated the benefits of improving depression symptoms. ${ }^{14,15,20,40}$ Among the probiotics evaluated, Bifidobacterium, Lactobacillus, and Lactococcus have displayed the ability to improve anxiety and depression symptoms. ${ }^{41-44}$ The mechanism of such psychobiotic strains could be explained by the combination of different protective mechanisms, including enhancing the barrier of the intestinal epithelium, inhibiting mast cell activation, stimulating antiinflammatory cytokines, and reducing lipopolysaccharide levels - that have been shown to play a role in the pathogenesis of depression and other mental illnesses. ${ }^{21}$ However, current research has not yet elucidated the interactions between the microbiome and the host, as well as the mechanisms of these interactions. The omics technologies, such as proteomics and metabolomics, might provide more insights into this novel field. $^{45}$
Despite optimistic findings in the role of probiotics in reducing the symptoms of mental disorder, ${ }^{14,40,46}$ a metaanalysis that pooled the results from 10 clinical trials showed no significant difference between patients who used probiotics and placebo. ${ }^{15}$ This could be explained by the differences in probiotic strains, dosage, and treatment duration, which leads to heterogeneity in the findings. More clinical trials need to be performed on particular probiotic strains with consistent doses and duration.

Patients with functional GI disorders were reported to have a lower quality of life than healthy controls. ${ }^{47}$ Therefore, in this study, we also assessed the quality of life using the EQ-5D-5L. ${ }^{32}$ Quality of life improved significantly at both 1 and 2 months. Improvements in the quality of life of IBS patients after using probiotics were also demonstrated in the studies by Pinto-Sanchez et al (using the SF36 scale) ${ }^{39}$ and Lorenzo-Zuniga et al (using 
the IBS-QoL scale). ${ }^{22}$ Decreased quality of life can be explained by the severity of the GI condition and psychological disturbances. ${ }^{48}$ Therefore, improving the quality of life in patients with functional GI disorders or chronic GI symptoms should be considered a treatment goal. ${ }^{48}$

The safety profile of the multispecies probiotic product cannot be established given the nature of the uncontrolled design of our study; therefore, we only recorded symptoms that developed while patients were using the product. Only low incidences of GI symptoms (heartburn, fullness, nausea, diarrhoea, and abdominal pain) and constitutional symptoms (sleeping complaints, headache, and dizziness) were reported, and these symptoms usually developed in the first week and resolved after 2 months. Even when all these symptoms were attributable to the probiotics product, our results remained consistent with the 2011 Agency for Healthcare Research and Quality report on the possible side effects of probiotics on the GI tract, such as abdominal pain, nausea, loose stools, and fullness. ${ }^{49,50}$ However, one patient developed a burning sensation in the throat 1 week after probiotic supplementation, which forced her to stop using the product after the first month then her sensation disappeared. While current evidence generally does not suggest an increased risk when using probiotics, there have been reported that immunocompromised patients might be more likely to experience adverse outcomes associated with probiotics. $^{51}$ More research using systematic reporting of adverse events needs to be done to establish the safety profile of probiotics.

This study has some important limitations. Firstly, the study was subject to common types of bias of an uncontrolled trial (eg, ascertainment bias, placebo response bias). Therefore, we could not make any inferences of associations regarding the effectiveness and safety of the product. Secondly, in this study, we did not collect sufficient data to analyze the effect of multiple factors on the change in patient's anxiety and depression symptoms. Thirdly, because of the short follow-up time, we could not evaluate the possible long-term effects of multispecies probiotics. However, the significant improvement in the first month and the continuous improvement in the second month after using the product suggest the need for randomized controlled trials on patients with chronic GI symptoms who present with anxiety and depression.

\section{Conclusion}

After 2 months of using the multispecies probiotics, anxiety and depression symptoms significantly improved in patients with chronic gastrointestinal symptoms. Mild gastrointestinal or constitutional symptoms developed in some patients.

\section{Acknowledgments}

We particularly thank Hoang Long Clinic (Vietnam) for agreeing to participate in this study. The probiotic product was sponsored by Winclove B.V Company and Dong Do Pharmacy Company. The original data of this study is shared by both the Institute of Gastroenterology and Hepatology and Winclove B.V Company. Any request on data sharing must be approved by both parties.

\section{Disclosure}

The authors declare that the research was conducted in the absence of any commercial or financial relationships that could be construed as a potential conflict of interest.

\section{References}

1. Fond G, Loundou A, Hamdani N, et al. Anxiety and depression comorbidities in irritable bowel syndrome (IBS): a systematic review and meta-analysis. Eur Arch Psychiatry Clin Neurosci. 2014;264:651-660. doi:10.1007/s00406-014-0502-z

2. Lee C, Doo E, Choi JM, et al. The increased level of depression and anxiety in irritable bowel syndrome patients compared with healthy controls: systematic review and meta-analysis. $J$ Neurogastroenterol Motil. 2017;23(3):349-362. doi:10.5056/jnm16220

3. Zhang A-Z, Wang Q-C, Huang K-M, et al. Prevalence of depression and anxiety in patients with chronic digestive system diseases: a multicenter epidemiological study. World $J$ Gastroenterol. 2016;22(42):9437-9444. doi:10.3748/wjg.v22.i42.9437

4. Van Oudenhove L, Crowell MD, Drossman DA, et al. Biopsychosocial aspects of functional gastrointestinal disorders. Gastroenterology. 2016;150:1355-1367.e2. doi:10.1053/j. gastro.2016.02.027

5. Wang H-X, Wang Y-P. Gut microbiota-brain axis. Chin Med J (Engl). 2016;129(19):2373-2380. doi:10.4103/0366-6999.190667

6. Cryan JF, Dinan TG. Mind-altering microorganisms: the impact of the gut microbiota on brain and behaviour. Nat Rev Neurosci. 2012;13(10):701-712. doi:10.1038/nrn3346

7. Schmidt C. Mental health: thinking from the gut. Nature. 2015;518 (7540):S12-15. doi:10.1038/518S13a

8. Amon P, Sanderson I. What is the microbiome? Arch Dis Child. 2017;102:edpract-2016.

9. Dinan TG, Cryan JF. Melancholic microbes: a link between gut microbiota and depression? Neurogastroenterol Motil. 2013;25 (9):713-719. doi:10.1111/nmo.12198

10. Ng QX, Soh AYS, Loke W, Lim DY, Yeo WS. The role of inflammation in irritable bowel syndrome (IBS). J Inflamm Res. 2018;11:345-349. doi:10.2147/JIR.S174982

11. Chong PP, Chin VK, Looi CY, Wong WF, Madhavan P, Yong VC. The microbiome and irritable bowel syndrome - a review on the pathophysiology, current research and future therapy. Front Microbiol. 2019;10:1136. doi:10.3389/fmicb.2019.01136

12. Grenham S, Clarke G, Cryan JF, Dinan TG. Brain-gut-microbe communication in health and disease. Front Physiol. 2011;2:94. doi:10.3389/fphys.2011.00094

13. Dinan TG, Stanton C, Cryan JF. Psychobiotics: a novel class of psychotropic. Biol Psychiatry. 2013;74(10):720-726. doi:10.1016/j. biopsych.2013.05.001 
14. Huang $\mathrm{R}$, Wang $\mathrm{K}$, Hu J. Effect of probiotics on depression: a systematic review and meta-analysis of randomized controlled trials. Nutrients. 2016;8(8):483. doi:10.3390/nu8080483

15. Ng QX, Peters C, Ho CYX, Lim DY, Yeo WS. A meta-analysis of the use of probiotics to alleviate depressive symptoms. J Affect Disord. 2018;228:13-19. doi:10.1016/j.jad.2017.11.063

16. Hotel A, Cordoba A. Health and nutritional properties of probiotics in food including powder milk with live lactic acid bacteria - joint FAO/ WHO expert consultation. Prevention. 2001;2014.

17. Morelli L, Capurso L. FAO/WHO guidelines on probiotics: 10 years later. J Clin Gastroenterol. 2012;46(Suppl):S1-S2. doi:10.1097/ MCG.0b013e318269fdd5

18. Morkl S, Butler MI, Holl A, Cryan JF, Dinan TG. Probiotics and the microbiota-gut-brain axis: focus on psychiatry. Curr Nutr Rep. 2020;9(3):171-182. doi:10.1007/s13668-020-00313-5

19. Tremblay A, Lingrand L, Maillard M, Feuz B, Tompkins TA. The effects of psychobiotics on the microbiota-gut-brain axis in early-life stress and neuropsychiatric disorders. Prog Neuropsychopharmacol Biol Psychiatry. 2021;105:110142. doi:10.1016/j.pnpbp.2020.110142

20. Steenbergen L, Sellaro R, van Hemert S, Bosch JA, Colzato LS. A randomized controlled trial to test the effect of multispecies probiotics on cognitive reactivity to sad mood. Brain Behav Immun. 2015;48:258-264. doi:10.1016/j.bbi.2015.04.003

21. Van Hemert S, Ormel G. Influence of the multispecies probiotic ecologic $^{\circledR}$ BARRIER on parameters of intestinal barrier function. Food Nutr Sci. 2014;5:1739-1745. doi:10.4236/fns.2014.518187

22. Lorenzo-Zuniga V, Llop E, Suarez C, et al. I.31, a new combination of probiotics, improves irritable bowel syndrome-related quality of life. World J Gastroenterol. 2014;20(26):8709-8716. doi:10.3748/ wjg.v20.i26.8709

23. Vuong DA, Van Ginneken E, Morris J, Ha ST, Busse R. Mental health in Vietnam: burden of disease and availability of services. Asian J Psychiatr. 2011;4(1):65-70. doi:10.1016/j.ajp.2011.01.005

24. Vu HTT, Lin V, Pham T, et al. Determining risk for depression among older people residing in Vietnamese rural settings. Int J Environ Res Public Health. 2019;16(15):2654. doi:10.3390/ijerph16152654

25. Vu HTT, Nguyen TX, Nguyen HTT, et al. Depressive symptoms among elderly diabetic patients in Vietnam. Diabetes Metab Syndr Obes. 2018;11:659-665. doi:10.2147/DMSO.S179071

26. Vu TH, Thu PH, Thien LC, Tuan NV, Hung NV. Characteristics and related factors of anxiety disorders in rheumatoid arthritis. Vietnam Med J. 2019;4:80-86.

27. Vo -T-T-K, Duy Thong V, Quynh B, Nguyen TM. Prevalence and dietary risk factors of irritable bowel syndrome in Vietnamese pharmacy students. Vietnam J Med Pharm. 2016;8:52-59. doi:10.34071/ jmp.2015.6.8

28. Snaith RP. The hospital anxiety and depression scale. Health Qual Life Outcomes. 2003;1:29. doi:10.1186/1477-7525-1-29

29. Herrmann C. International experiences with the hospital anxiety and depression scale-a review of validation data and clinical results. J Psychosom Res. 1997;42(1):17-41. doi:10.1016/S0022-3999(96) 00216-4

30. Olsson I, Mykletun A, Dahl AA. The hospital anxiety and depression rating scale: a cross-sectional study of psychometrics and case finding abilities in general practice. BMC Psychiatr. 2005;5:46. doi:10.1186/1471-244X-5-46

31. Wu Y, Levis B, Sun Y, et al. Accuracy of the hospital anxiety and depression scale depression subscale (HADS-D) to screen for major depression: systematic review and individual participant data meta-analysis. BMJ. 2021;373:n972. doi:10.1136/bmj.n972

32. Mai VQ, Sun S, Minh HV, et al. An EQ-5D-5L value set for Vietnam. Qual Life Res. 2020;29:1923-1933. doi:10.1007/s11136-020-02469-7

33. EuroQol Research Foundation. EQ-5D-5L; 2009. Available from: https://euroqol.org/eq-5d-instruments/eq-5d-5l-about/. Accessed June $2,2021$.
34. Hartono JL, Mahadeva S, Goh KL. Anxiety and depression in various functional gastrointestinal disorders: do differences exist? $J \quad$ Dig Dis. 2012;13(5):252-257. doi:10.1111/j.17512980.2012.00581.x

35. Shah E, Rezaie A, Riddle M, Pimentel M. Psychological disorders in gastrointestinal disease: epiphenomenon, cause or consequence? Ann Gastroenterol. 2014;27(3):224-230.

36. Phuc NN, Quynh BTH, Thao DN, Thong VD. Reliability of the Vietnamese version of irritable bowel syndrome quality of life. Ho Chi Minh City J Med. 2018;22(5):396-399.

37. Chahwan B, Kwan S, Isik A, van Hemert S, Burke C, Roberts L. Gut feelings: a randomised, triple-blind, placebo-controlled trial of probiotics for depressive symptoms. J Affect Disord. 2019;253:317-326. doi:10.1016/j.jad.2019.04.097

38. Bagga D, Aigner CS, Reichert JL, et al. Influence of 4-week multi-strain probiotic administration on resting-state functional connectivity in healthy volunteers. Eur J Nutr. 2019;58(5):1821-1827. doi:10.1007/s00394-018-1732-z

39. Pinto-Sanchez MI, Hall GB, Ghajar K, et al. Probiotic bifidobacterium longum NCC3001 reduces depression scores and alters brain activity: a pilot study in patients with irritable bowel syndrome. Gastroenterology. 2017;153(2):448-459.e448. doi:10.1053/j. gastro.2017.05.003

40. Wallace CJK, Milev R. The effects of probiotics on depressive symptoms in humans: a systematic review. Ann Gen Psychiatry. 2017;16:14. doi:10.1186/s12991-017-0138-2

41. Benton D, Williams C, Brown A. Impact of consuming a milk drink containing a probiotic on mood and cognition. Eur J Clin Nutr. 2007;61(3):355-361. doi:10.1038/sj.ejcn.1602546

42. Rao AV, Bested AC, Beaulne TM, et al. A randomized, double-blind, placebo-controlled pilot study of a probiotic in emotional symptoms of chronic fatigue syndrome. Gut Pathog. 2009;1(1):6. doi:10.1186/ 1757-4749-1-6

43. Yamamura S, Morishima H, Kumano-go T, et al. The effect of Lactobacillus helveticus fermented milk on sleep and health perception in elderly subjects. Eur J Clin Nutr. 2009;63(1):100-105. doi:10.1038/sj.ejen.1602898

44. Messaoudi M, Violle N, Bisson J-F, Desor D, Javelot H, Rougeot C. Beneficial psychological effects of a probiotic formulation (Lactobacillus helveticus R0052 and Bifidobacterium longum R0175) in healthy human volunteers. Gut Microbes. 2011;2 (4):256-261. doi:10.4161/gmic.2.4.16108

45. Wang P, Ng QX, Zhang H, Zhang B, Ong CN, He Y. Metabolite changes behind faster growth and less reproduction of Daphnia similis exposed to low-dose silver nanoparticles. Ecotoxicol Environ Saf. 2018;163:266-273. doi:10.1016/j.ecoenv.2018.07.080

46. Noonan S, Zaveri M, Macaninch E, Martyn K. Food \& mood: a review of supplementary prebiotic and probiotic interventions in the treatment of anxiety and depression in adults. BMJ Nutr Prev Health. 2020;3:351-362. doi:10.1136/bmjnph-2019-000053

47. Choi MG, Jung HK. Health related quality of life in functional gastrointestinal disorders in Asia. J Neurogastroenterol Motil. 2011;17(3):245-251. doi:10.5056/jnm.2011.17.3.245

48. Simren M, Svedlund J, Posserud I, Bjornsson ES, Abrahamsson H. Health-related quality of life in patients attending a gastroenterology outpatient clinic: functional disorders versus organic diseases. Clin Gastroenterol Hepatol. 2006;4(2):187-195. doi:10.1016/S15423565(05)00981-X

49. Didari T, Solki S, Mozaffari S, Nikfar S, Abdollahi M. A systematic review of the safety of probiotics. Expert Opin Drug Saf. 2014;13 (2):227-239. doi:10.1517/14740338.2014.872627

50. Doron S, Snydman DR. Risk and safety of probiotics. Clin Infect Dis. 2015;60(Supp12):S129-S134. doi:10.1093/cid/civ085

51. Hempel S, Newberry S, Ruelaz A, et al. Safety of probiotics used to reduce risk and prevent or treat disease. Evid Rep Technol Assess (Full Rep). 2011;(200):1-645. 


\section{Publish your work in this journal}

The Journal of Multidisciplinary Healthcare is an international, peerreviewed open-access journal that aims to represent and publish research in healthcare areas delivered by practitioners of different disciplines. This includes studies and reviews conducted by multidisciplinary teams as well as research which evaluates the results or conduct of such teams or healthcare processes in general. The journal covers a very wide range of areas and welcomes submissions from practitioners at all levels, from all over the world. The manuscript management system is completely online and includes a very quick and fair peer-review system. Visit http://www.dovepress.com/testimonials. php to read real quotes from published authors. 